\title{
Investigation of 2D-WH/TS OCDMA System Performance under the Influence of PMD
}

\author{
Ivan Glesk, Senior Member, IEEE, Saleh Seyedzadeh, Jozef Dubovan*, Milan Dado*, \\ and Wing C. Kwong**, Senior Member, IEEE
}

\author{
Faculty of Engineering, University of Strathclyde, Glasgow G1 1XW, UK \\ * Faculty of Engineering, University of Žilina, Žilina 010 26, SK \\ ** Department of Engineering, Hofstra University, Hempstead, USA \\ Tel: +44 (141) 548 2529, e-mail: ivan.glesk@strath.ac.uk
}

\begin{abstract}
The impact of a differential group delay (DGD) and polarization mode dispersion (PMD) on multi-wavelength picosecond code carriers used by two-dimensional wavelength-hopping time-spreading coding schemes in optical code division multiple access (2D-WH/TS OCDMA systems is investigated. Based on experimental data collected over ten days on a $111 \mathrm{~km}$ long commercially used fiber link PMD and DGD parameters are determined and used to find the code carriers temporal broadening. This information is then used to calculate the 2D-WH/TS OCDMA system performance degradation as a function of varying number of simultaneous users for different DGD.
\end{abstract}

Keywords: OCDMA, polarization mode dispersion, differential-group delay, code carriers broadening

\section{INTRODUCTION}

Optical code-division multiple access (OCDMA) is an attractive multiplexing technique offering a dynamic bandwidth allocation, efficiency in a bursty traffic, a soft limit on a number of simultaneous users, and the improved privacy [1-3]. With the advent of modern optical devices, a high capacity OCDMA system operating at $320 \mathrm{Gbit} / \mathrm{s}(32 \times 10 \mathrm{Gbit} / \mathrm{s})$ has been demonstrated [4]. High performance OCDMA systems often utilise TwoDimensional Wavelength-Hopping Time-Spreading (2D-WH/TS) family of codes based on multi-wavelength picosecond code carriers [5] by spreading them in wavelength and time domains. When such codes are transmitted, it is essential to maintain the carriers' temporal shape to ensure the highest possible OCDMA system performance. Extensive research has been done to investigate the effect of fibre impairments such as chromatic dispersion, temperature-induced dispersion and the impact of the chirp on the OCDMA performance [6 - 10]. The main goal was to understand any system performance degradation resulted from the temporal code carriers broadening.

This paper investigates the impact of a differential-group delay (DGD) on the family of 2D-WH/TS codes based on multi-wavelength picosecond code carriers and the resulted system performance degradation.

\section{OPTICAL PULSE BROADENING INDUCED BY PMD}

Sunnerud, et al. [11] developed a comprehensive theory about PMD effects on the fibre transmitted signal. This applies to arbitrary wavelength dependencies on chromatic dispersion (CD), chirp, and polarization mode dispersion (PMD) of random orders. For a Gaussian pulse shape, the average output pulse width $(\tau)$ and its root means square (RMS) value affected by an uncompensated fiber transmission system could be expressed as:

$$
\tau=E\left\{\tau^{2}\right\}=\tau_{0}^{2}+\frac{E\left\{\Delta \tau^{2}\right\}}{4}-\frac{\tau_{0}^{2}}{2}\left(\sqrt{1+\frac{E\left\{\Delta \tau^{2}\right\}}{3 \tau_{0}^{2}}}-1\right)
$$

Where $\tau_{0}$ denotes the RMS pulse width at the fibre link input and $\Delta \tau$ is a value of DGD. Figure 1 illustrates

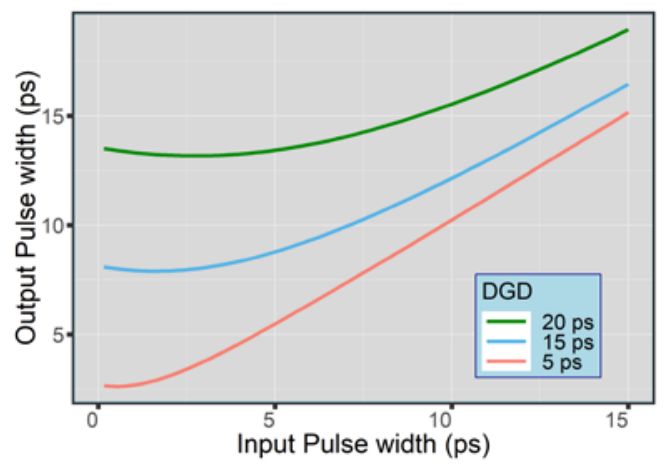

Figure 1. RMS output vs. input pulse width for the different DGD values. 
a calculated temporal output pulse width $(\tau)$ versus the input pulse width $\left(\tau_{0}\right)$ for different values of DGD.

\section{EXPERIMENTAL EVALUATION OF FIBER LINK PMD AND DGD}

Figure 2 shows an experimental setup used to measure a time-dependent DGD which is the key factor influencing the PMD.

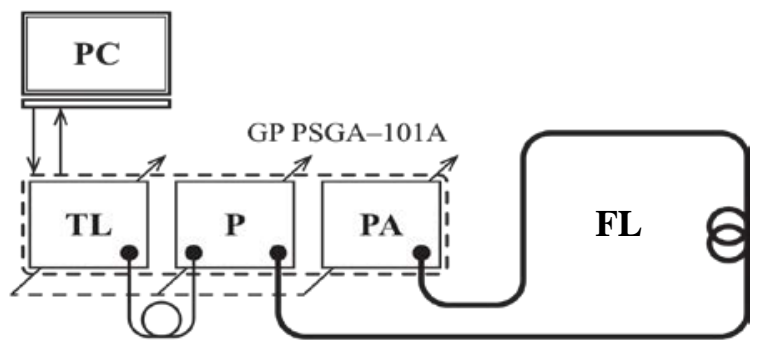

Figure 2. Experimental setup for measuring DGD of fibre link FL by using General Photonics Polarization Measurement System GP PSGA-101A. TL - tuneable laser, P - polarizer, PA - polarization analyser. Fiber type used by the link was ITU-T G.652.

The fiber link (FL) under the test was part of a commercially deployed cable system composed of an electrical grounding wire wrapped around the fiber link and consisted of two main sections (see Fig. 3): Sections shown by a dotted line were under the ground and had a length of $4 \mathrm{~km}$ and $0.5 \mathrm{~km}$, respectively. The section of $51 \mathrm{~km}$ (shown by a solid line) was above the ground. At its right end (see Fig. 3), the fiber link was looped-back, thus providing a $111 \mathrm{~km}$ long roundtrip when viewed from the point of measurements (M).

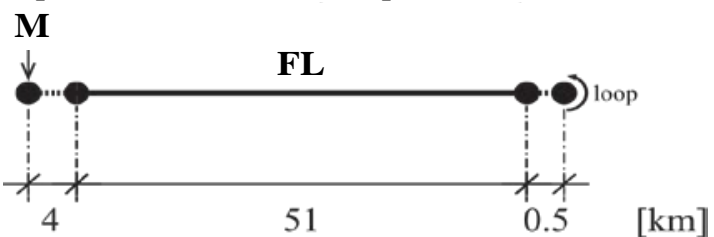

Figure 3. Schematic diagram of a fibre link loop under the test. The dotted line is its underground section, whereas the solid line represents the section above the ground. $M$ indicates the point from where the measurements were conducted.

All measurements were carried out from the location marked (M) by using General Photonics PSGA- 101A. Each measurement cycle was done over a wavelength range from $1528.97 \mathrm{~nm}$ to $1563.66 \mathrm{~nm}$ with a $50 \mathrm{GHz}$ step (i.e., 88 wavelengths in total). We used the Jones matrix eigen-analysis method. Measurements took place from $10^{\text {th }}$ April to $19^{\text {th }}$ April 2019 and were repeated every 60 seconds. During each measurement, the values of PMS, DGD, second-order DGD, principal state of polarization, and polarization dependent loss were recorded. For an illustration, for each measurement cycle, a DGD value was calculated as the average value for all 88 wavelengths. Obtained values were then plotted in Fig. 4 to illustrate how the average DGD value (AVG DGD) changes with time in this wavelength region. The max and min value of 3,86 and 0,524 ps, respectively can be noted.

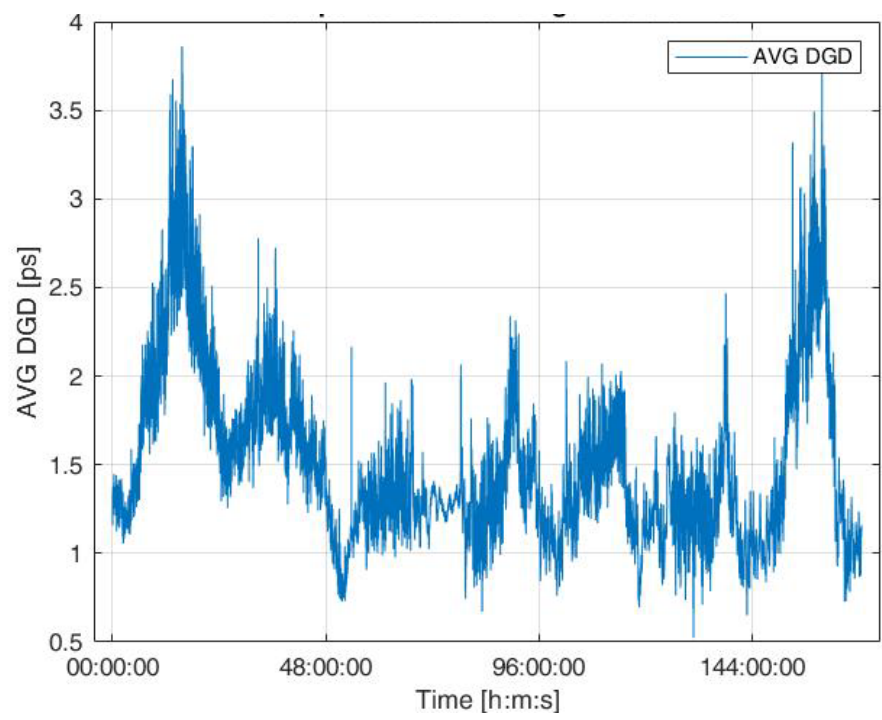

Figure 4. Time dependency of fiber link average DGD value in the spectral region (1528.97- 1563.66) $\mathrm{nm}$. 


\section{EFFECT OF DGD ON 2D-WH/TS OCDMA SYSTEM PERFORMANCE}

The performance of a 2D-WH/TS OCDMA system can be described by a probability of error $\left(P_{\mathrm{e}}\right)$ as a function of a number of simultaneous users $(K)$. For an OCDMA receiver with hard limiting capabilities [5]

$$
P_{e}=\frac{1}{2} \sum_{i=0}^{w}(-1)^{w-i} \frac{w !}{[i !(w-i) !]}\left(q_{0}+i q_{1} \frac{1}{w}\right)^{k-1}
$$

where $q_{0}=1-q_{1}$ and $q_{1}=\frac{w^{2}}{2 N L}$. Here, $w$ is a code weight, $L$ is a number of used wavelength code carriers. Depending on a 2D-WH/TS OCDMA system design, the number of chips available to host the wavelength code carriers will be $N=T / \tau_{0}$. However, due to the presence of DGD, the code carriers will undergo a temporal broadening given by (1). As a consequence, the number of chips $N$ will decrease to a smaller value of $T / \tau$.

The instantaneous DGD in the (1538.0 - 1540.8) nm region can be seen in Fig. 5. In order to investigate its impact

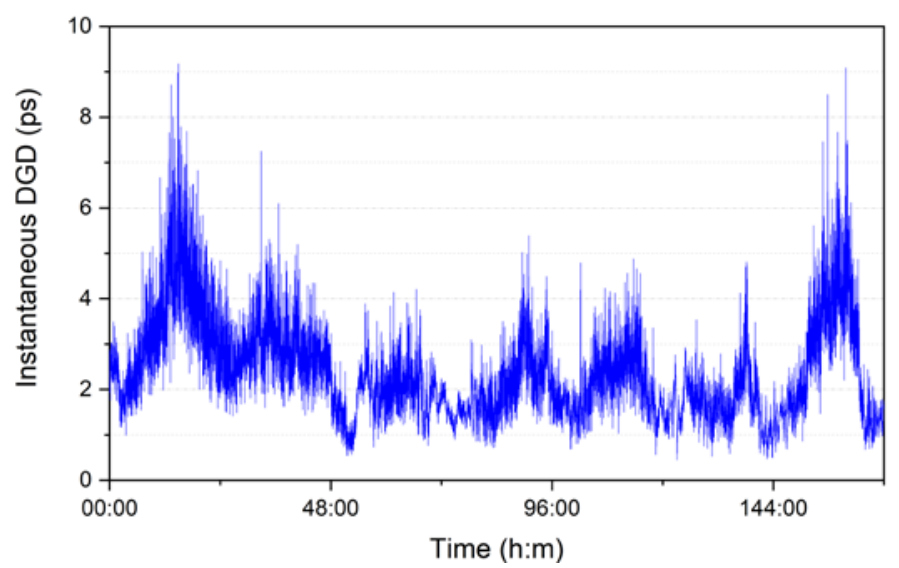

Figure 5. Instantaneous values of measured DGD.

on a 2D-WH/TS OCDMA, the system was designed based on $L=8$ code carriers $\lambda_{1}=1538.0 \mathrm{~nm}, \lambda_{2}=1538.4 \mathrm{~nm}$, $\lambda_{3}=1538.8 \mathrm{~nm}, \lambda_{4}=1539.2 \mathrm{~nm}, \lambda_{5}=1539.6 \mathrm{~nm}, \lambda_{6}=1540 \mathrm{~nm}, \lambda_{7}=1540.4 \mathrm{~nm}$, and $\lambda_{8}=1540.8 \mathrm{~nm}$ having a $50 \mathrm{GHz}(0.4 \mathrm{~nm})$ spectral spacing. Assuming a temporal input pulse width of $\tau_{0}=4 \mathrm{ps}$ for all wavelength code carriers, based on the measurements and using (1) their relative temporal broadening as a function of time varying DGD was then calculated. The obtained results are shown in Fig. 6.

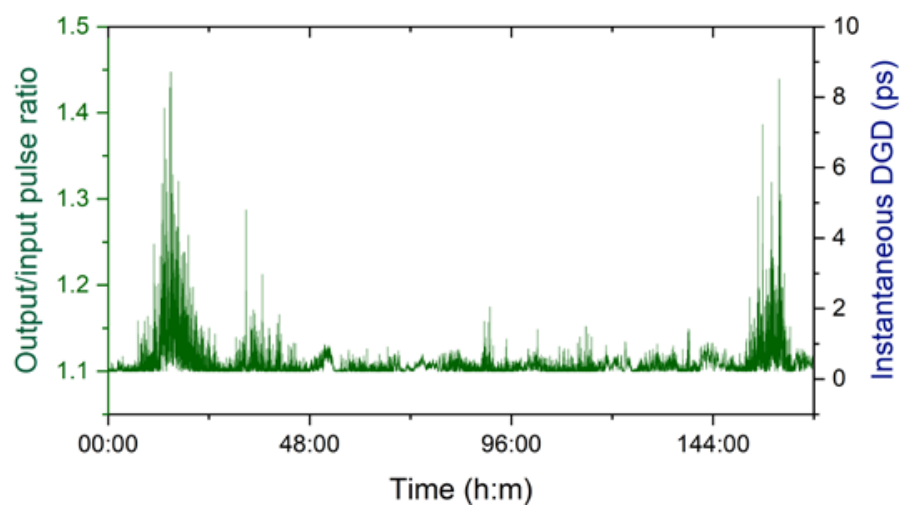

Figure 6. Illustration of the impact of fibre link DGD fluctuations on the code carriers' temporal width in (1538.0 -1540.8) nm spectral region. The output /input temporal width ratio is defined as $\tau / \tau_{0} . \tau_{o}=4 \mathrm{ps}$ is used as the temporal input width for all code carriers.

Based on the results in Fig. 6 and using (2) we then calculated the probability of error $\left(P_{e}\right)$ for the three different DGD values: $\mathrm{DGD}=0$, DGD $=9.17 \mathrm{ps}$ (the maximal instantaneous value measured), and DGD $=5$ ps (which is the upper limit for $97.5 \%$ of measured cases). The results are shown in Fig. 7. Here, by taking the advantage of OCDMA soft blocking capabilities, we first let the $P_{\mathrm{e}}$ value to deteriorate to $10^{-4}$ by adding more users, and in turn, we bring it back to $10^{-9}$ by using a forward error correction scheme. With this approach, the max number of simultaneous users can be increased to 90 for $\mathrm{DGD}=0$. When the max DGD $=9.17$ is present, the number of users drops to 60 . However, DGD smaller than 5 ps was found in $97.5 \%$ of measurements. Here, the OCDMA system will support up to 80 simultaneous users, just 10 users less than the case with DGD $=0$. 


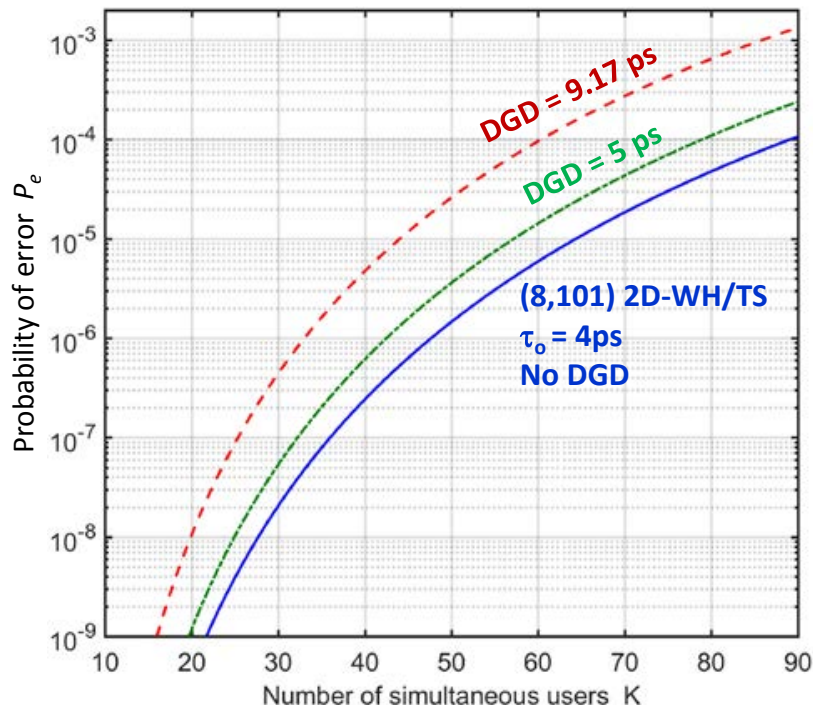

Figure 7. Performance degradation of 2D-WH/TS OCDMA system based on 8 multi-wavelengths 4 ps wide code carriers from $(1538.0$ - 1540.8) $\mathrm{nm}$ spectral region. Case of no DGD, DGD $=5$ ps and 9.17 ps, respectively.

\section{CONCLUSIONS}

The impact of a differential group delay on a $(8,101)$ 2D-WH/TS OCDMA system designed using 8 multiwavelength 4 ps wide code carriers over a $111 \mathrm{~km}$ long commercial fiber link was investigated. In a worst case scenario, when all 8 code carriers are from a spectral region with the highest found DGD of 9.17 ps, the max number of simultaneous users will drop by a 1/3, from 90 to 60 . However, $97.5 \%$ of the time, DGD was less than 5 ps and the system will support up to 80 simultaneous users. This is just 10 users less than the case with no DGD.

\section{ACKNOWLEDGEMENTS}

This work was supported in part by the Faulty Development and Research Grants of Hofstra University, in part by the European Union's Horizon 2020 research and innovation program under the Marie Sklodowska-Curie grant agreement No 734331.

\section{REFERENCES}

[1] I. Glesk, T. Osadola, W. C. Kwong, “Towards higher scalability of hybrid optical CDMA network,” Opt. Quant. Electron. 49:267 (2017). DOI 10.1007/s11082-017-1101-9.

[2] S. Seyedzadeh, F. P. Rahimian, I. Glesk, and M. H. Kakaee, "Variable weight spectral amplitude coding for multiservice OCDMA networks,” Opt. Fiber Technol., vol. 37, pp. 53-60, 2017.

[3] I. Glesk, Y.-K. Huang, K. S. Kravtsov, and P. R. Prucnal, "Increasing optical security with OCDMA using optical XOR,” In: 2007 Photonics in Switching, pp. 105-106, 2007. DOI: 10.1109/PS.2007.4300766.

[4] V. J. Hernandez et al., “A 320-Gb/s capacity (32-User × 10 Gb/s) SPECTS O-CDMA network testbed with enhanced spectral efficiency through forward error correction,” J. Light. Technol., vol. 25, no. 1, pp. 7986, 2007. DOI: 10.1109/JLT.2006.888927.

[5] W. C. Kwong and G.-C. Yang, Optical Coding Theory with Prime, New York: CRC Press, 2013.

[6] Md. S. Ahmed and I. Glesk, “Application of Semiconductor Optical Amplifier (SOA) in Managing Chirp of Optical Code Division Multiple Access (OCDMA) Code Carriers in Temperature Affected Fibre Link,” Appl. Sci., 8 (5), 715 (2018). DOI: https://doi.org/10.3390/app8050715.

[7] Md. S. Ahmed and I. Glesk, "Management of OCDMA Auto-correlation Width by Chirp Manipulation Using SOA,” IEEE Photon. Technol. Lett. vol. 30, no. 9, pp. 785-789 (May 1, 2018).

DOI: 10.1109/LPT.2018.2814788.

[8] Md S. Ahmed and I. Glesk, "Mitigation of Temperature Induced Dispersion in Optical Fiber on OCDMA Auto-correlation,” IEEE Photon. Technol. Lett., vol. 29, no. 22, pp. 1979-1982 (Nov 15, 2017). DOI 10.1109/LPT.2017.2758163.

[9] T. B. Osadola, S. K. Idris, I. Glesk, W. C. Kwong, "Effect of Environmental Variations in Temperature on 2D-WH/TS OCDMA Code Performance,” Journal of Optical Communications and Networking 5 (1), pp. 68-73 (2013). http://dx.doi.org/10.1364/JOCN.5.000068.

[10] Z. Pan, C. Yu, and A. E. Willner, "Optical performance monitoring for the next generation optical communication networks,” Opt. Fiber Technol., vol. 16, no. 1, pp. 20-45, 2010.

[11] H. Sunnerud, M. Karlsson, C. Xie, and P. A. Andrekson, "Polarization-mode dispersion in high-speed fiber-optic transmission systems,” J. Light. Technol., vol. 20, no. 12, pp. 2204-2219, Dec. 2002. 